\title{
O PROBLEMA DOS PONDERADORES NO ENSINO DA ESTATÍSTICA
}

\section{THE PROBLEM OF WEIGHTS IN TEACHING STATISTICS}

\author{
Valéria Guimarães Moreira ${ }^{1}$ \\ Guilherme Guimarães Moreira ${ }^{2}$
}

\begin{abstract}
Resumo: Este artigo $^{3}$ apresenta uma proposta para o ensino das quantidades estatísticas com o uso de ponderadores de forma mais enfática na Educação Básica, baseando-se nas experiências docentes e profissionais dos autores. Observamos que, geralmente, é despendido muito tempo no ensino da estatística com estimadores pontuais como a média, entre outras, porém sem o uso de pesos amostrais. Isto acarreta uma dificuldade no aluno nos anos finais do ensino médio e, ou, no ensino superior ou em ambientes de trabalho mais tarde, a trabalharem com ponderadores. Porém, no trabalho da Estatística no mundo real é imprescindível o uso destes.
\end{abstract}

Palavras - chave: Educação Estatística; Educação Básica; Ponderadores.

Abstract: This paper presents a proposal for high school filling with the use of more emphatic weights in Basic Education, based on the teaching and professional experiences of authors. Note that a lot of time is usually spent teaching statistics with point estimates as average, among others, but without the use of sample weights. That is, it is not a means of education without students in the final years of high school and, in higher education, or in later work environments, a weighted worker. However, no work of statistics in the world is essential to use them.

Keywords: Statistical Education; Basic Education; Weightings.

\section{Introdução}

O ensino da estatística na contemporaneidade se tornou essencial para que o cidadão possa exercer de forma plena a sua cidadania, pois fornece as ferramentas necessárias à obtenção, construção e interpretação corretas das informações do cotidiano. A todo o momento o cidadão recebe informações sociais, econômicas e ambientais que impactam no seu dia a dia: índices de inflação e desemprego, expectativas de aumento da taxa de juros, geadas e secas que impactam no preço dos alimentos, etc. E apenas o

\footnotetext{
${ }^{1}$ Doutorado em Ensino de Ciências e Matemática pela Universidade Cruzeiro do Sul (UNICSUL). Centro Federal de Educação Tecnológica de Minas Gerais (CEFET-MG), Belo Horizonte, M inas Gerais, Brasil. E-mail: valeriagm@cefetmg.br

${ }^{2}$ Mestrado em Estatística pela Universidade Federal de Minas Gerais (UFMG). Instituto Brasileiro de Geografia e Estatística (IBGE), Belo Horizonte, Minas Gerais, Brasil. E-mail: guilherme.moreira@ibge.gov.br

3 O IBGE se isenta de qualquer responsabilidade pelas opiniões, informações, dados e conceitos, apresentados neste artigo e que são de inteira e exclusiva responsabilidade dos autores.
} 
DOI: http://dx.doi.org/10.33238/ReBECEM.2019.v.3.n.2.22605

tratamento adequado da informação possibilitará ao cidadão uma melhor interpretação do ambiente que o cerca, podendo inclusive gerar uma melhor tomada de decisão.

Portanto, o ensino da estatística se torna essencial no processo de aprendizagem e formação dos alunos em todos os níveis de ensino: fundamental, médio e graduação. Esse tema está previsto na educação básica no ensino de matemática como Tratamento da Informação no Ensino Fundamental e Análise de Dados no Ensino Médio, segundo os PCN's nacionais.

A Estatística deve ser vista, então, como um conjunto de ideias e procedimentos que permitem aplicar a Matemática em questões do mundo real, mais especialmente, aquelas provenientes de outras áreas. Devem ser vistas também como formas de a Matemática quantificar e interpretar conjuntos de dados ou informações que não podem ser quantificados direta ou exatamente. Cabe à Estatística, por exemplo, analisar a intenção de voto em uma eleição ou o possível êxito do lançamento de um produto no mercado, antes da eleição em si e da fabricação do produto. Isso é feito através da pesquisa estatística, que envolve amostras, levantamento de dados e análise das informações obtidas (BRASIL, 2006, p.126).

Durante o ensino fundamental, a construção das quantidades estatísticas de interesse é baseada em populações muito pequenas. Porém, no ensino médio e na graduação, as populações de interesse são conjuntos imensos de indivíduos, o que faz com que estas estatísticas sejam construídas a partir de amostras.

No caso de estatísticas de interesse oriundas de amostras, cada unidade da amostra em questão representa um número de unidades da população. Surge, então, a necessidade de se trabalhar com ponderadores para se estimar os parâmetros populacionais de interesse. Em estatística estes ponderadores são denominados pesos amostrais.

Sua construção está associada à fase de aleatorização e seleção das unidades amostrais que comporão a amostra a ser analisada e/ou estudada. Este pequeno conjunto é de suma importância para o estudo, pois podemos generalizar os resultados encontrados no estudo desta amostra para todo o universo de interesse, e isto, só se torna possível por conta dos ponderadores.

\section{0 problema dos ponderadores}

Um dos problemas no ensino da Estatística é o "medo" de se trabalhar com ponderadores, seja porque se considere difícil o cálculo, seja porque a conta fica mais trabalhosa. Um dos motivos que gera essa dificuldade pode ser porque, comumente, se apresenta uma forma de cálculo simplificada para as quantidades estatísticas e se gasta muito tempo, na educação básica, focado em problemas simples e não corriqueiros os 
DOI: http://dx.doi.org/10.33238/ReBECEM.2019.v.3.n.2.22605

quais somente com o uso da fórmula se chega ao resultado, sem que haja um esforço de raciocínio no qual se precisem conhecer as parcelas da operação, enxergá-las no contexto e utilizá-las de forma adequada a se construir a estatística de interesse.

Onde enxergamos esse problema

Por exemplo, a média, $\bar{x}$, é uma das estatísticas de interesse mais utilizadas. Ela é o resultado da divisão de um total estatístico de uma variável de interesse pela quantidade de unidades que foram necessárias para se calcular aquele total. Desta forma temos que a fórmula da média é:

$$
\bar{x}=\frac{T}{N}
$$

onde, $T$ é o total da variável de interesse e $N$ a quantidade de unidades da população de interesse.

Conceitualmente esta é a definição de média. Porém, a sua expressão matemática mais utilizada é aquela em que os registros amostrais aparecem, neste caso sua expressão é assim definida:

$$
\bar{x}=\frac{\sum_{i}^{n}=1^{w_{i} x_{i}}}{\sum_{i}^{n}=1^{w_{i}}}
$$

onde, temos que o total $T$ passa a ser agora uma soma ponderada, onde cada registro da variável de interesse $x, x_{i}$ é multiplicada por um ponderador individualizado, $w_{i}$, o qual significa que o $i$-ésimo registro da amostra representa $w_{i}$, unidades da população de interesse.

Esta é a forma genérica de cálculo da média estatística, ela não é a média aritmética simples, pois a média aritmética simples, em Estatística, só é utilizada quando temos um recenseamento de unidades de uma população ou quando de uma determinada população se retira uma amostra aleatória simples. Nestes casos, temos o seguinte:

1. Em um recenseamento de unidades de uma população, como todas as unidades desta população serão investigadas, elas se autorrepresentam e, portanto, neste caso $w_{i}=1$. Fazendo com que a fórmula em questão esteja simplificada na forma da fórmula matemática da média aritmética simples.

2. No caso de uma amostra populacional ser uma amostra aleatória simples, todas as unidades terão a mesma chance de fazerem parte da amostra e, portanto, neste caso $w_{i}=w$. E novamente, conseguimos simplificar a fórmula em na forma da fórmula matemática da média aritmética simples. 
DOI: http://dx.doi.org/10.33238/ReBECEM.2019.v.3.n.2.22605

A partir de nossa experiência docente no ensino de estatística, seja na disciplina de matemática da Educação Básica, seja na graduação, percebemos que é comum utilizar exemplos triviais no ensino da Estatística na Educação Básica que dificilmente tem relação com problemas da vida real. Acreditamos que o ideal seria dedicar um tempo maior no ensino para a utilização de fórmulas ponderadas, de modo que o aluno construa habilidades lógico-cognitivo-matemáticas para se trabalhar com ponderadores.

Isto porque no mundo real não trabalhamos com censo de unidades, pois é muito caro e dispendioso, trabalhamos com amostras que quase sempre são estratificadas, ou conglomeradas, quando não estratificadas e conglomeradas ao mesmo tempo, reduzindo assim a incerteza e o custo do processo de conhecimento sobre um determinado fenômeno.

Pesquisas de órgãos produtores de informação como o IBGE, o Ministério da Saúde, a FioCruz, a Fundação João Pinheiro, FGV, e etc., tem-se que a grande maioria de suas estatísticas de interesse são baseadas em amostras probabilísticas estratificadas, conglomeradas ou estratificadas e conglomeradas, de forma que as médias apresentadas não podem ser médias aritméticas simples e sim médias aritméticas ponderadas a fim de que se obtenha estimadores não viciados destas estatísticas de interesse.

Podemos citar como fontes de informação que afetam o nosso dia-a-dia, as seguintes estatísticas de interesse, dentre outras:

1. Pesquisa Nacional de Amostra de Domicílios Contínua $(P N A D-C)^{4}$, do IBGE, que nos fornece dados demográficos sobre a população brasileira e a taxa de desemprego.

2. Pesquisa Econômicas Estuturais do Comércio $(P A C)^{5}$, Serviços $(P A S)^{6}$, da Indústria $(P I A)^{7}$ e da Indústria da Construção $(P A I C)^{8}$, do IBGE, que nos fornece dados econômicos de longo prazo (anual e série histórica) sobre o setores econômicos da economia brasileira, seus custos e despesas, pessoal ocupado, salários, receita dentre outras.

\footnotetext{
${ }^{4}$ IBGE. Pesquisa Nacional por Amostra de Domicílios Contínua: Notas Técnicas. Versão 1.5. 4. ed. Rio de Janeiro: IBGE, 2019 c.

${ }^{5}$ IBGE. Pesquisa Anual de Comércio 2016. Volume 28. Páginas 1 a 59. Rio de Janeiro: IBGE, 2018 a.

${ }^{6}$ IBGE. Pesquisa Anual de Serviços 2016. Volume 18. Páginas 1 a 56. Rio de Janeiro: IBGE, 2018 b.

${ }^{7}$ IBGE. Pesquisa Industrial 2017. Volume 36. Número 1. Páginas 1 a 45. Rio de Janeiro: IBGE, 2019 b.

${ }^{8}$ IBGE. Pesquisa Anual da Indústria da Construção 2017. Volume 27. Páginas 1 a 35 . Rio de Janeiro: IBGE, 2019 a.
} 
DOI: http://dx.doi.org/10.33238/ReBECEM.2019.v.3.n.2.22605

3. Pesquisa de Preços da Fundação Getúlio Vargas $(I G P-M)^{9}$, da FGV, que também calcula a inflação e é o principal índice de preços aplicado ao mercado nas transações entre empresas e sociedade, como por exemplo, o reajuste do aluguel.

4. Pesquisa de Saúde do Escolar (Pense $)^{10}$, do Ministério da Saúde, realizada com o apoio do IBGE e, que fornece dados sobre a saúde dos adolescentes para todo o território nacional, estados, capitais e interior e por redes de ensino, pública e privada.

5. Produto Interno Bruto de Minas Gerais ${ }^{11}$, da Fundação João Pinheiro, a qual em sua confecção utiliza as pesquisas econômicas conjunturais do IBGE: $\mathrm{PMS}^{12}$,

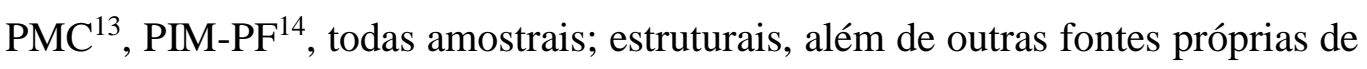
informação pertinentes ao Estado.

Este tipo de situação serve para qualquer estatística de interesse, seja ela um total, uma mediana, um quantil, uma variabilidade, um coeficiente de variação, um desvio padrão, dentre outros. Todas as estatísticas de interesse são sempre ponderadas como podemos ver, muitas das vezes, mesmo em recenseamentos há pesos de expansão por conta da não resposta de algumas unidades populacionais e, portanto, um ponderador passa a ser necessário para que a estimativa da estatística de interesse possa atingir, de forma não viesada, o parâmetro populacional de interesse.

Um dos equívocos mais comuns que observamos em nossa prática docente entre os alunos de graduação ocorre quando ao fazerem a modelagem de uma população a partir de uma amostra, eles não levam em conta os pesos amostrais em seu modelo acarretando distorções aos resultados dos modelos e por conseguinte na análise do problema. Acreditamos, a partir de nossa prática profissional, que esse equívoco é consequência na falta do uso de ponderadores, de forma mais enfática, com problemas mais relacionados a problemas de situações reais, durante o ensino de estatística, na Educação Básica.

\footnotetext{
9 FUndAÇÃO GETÚliO VARGAS. Índice Geral de Preços - Mercado (IGP - M): Metodologia. Páginas: 1 a 65. Rio de Janeiro: IBGE/FGV. 2016.

${ }^{10}$ IBGE, Pesquisa Nacional de Saúde do Escolar 2015. Páginas: 1 a 132. Rio de Janeiro: IBGE. 2016.

11 FUNDAÇÃO JOÃO PINHEIRO. Produto Interno Bruto de Minas Gerais 2016. Diretoria de Estatísticas e Informações. Série Indicadores Econômicos. N. 16. Páginas: 1 a 48. Belo Horizonte: FJP, 2018.

12 IBGE, Pesquisa Mensal de Serviços. Volume 42. Série Relatórios Metodológicos. Rio de Janeiro: IBGE, 2015b.

${ }^{13}$ IBGE, Pesquisa Mensal de Comércio. Volume 15. Série Relatórios Metodológicos. Edição $4^{\mathrm{a}}$. Rio de Janeiro: IBGE, 2015a.

14 IBGE, Indicadores Conjunturais da Indústria: Produção. Volume 31. Série Relatórios Metodológicos. 2. ed. Rio de Janeiro: IBGE, 2015 c.
} 


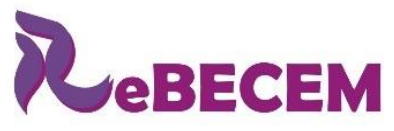

DOI: http://dx.doi.org/10.33238/ReBECEM.2019.v.3.n.2.22605
Revista Brasileira de Educação em

Ciências e Educação Matemática

Como podemos ver, o uso de ponderadores é uma dificuldade real apresentada pelos alunos no estudo estatístico, seja no nível médio, seja na graduação. Tal dificuldade acarreta ao aluno, em nossa visão, problemas em assimilar conceitos estatísticos relevantes e constrói uma dificuldade em se trabalhar habilidades estatísticas com clareza e destreza que nos são úteis em nossas vidas laborais.

\section{Pesquisas Científicas sobre o Ensino da Estatística}

Os autores Bianchini (2013) e Júnior e Vaz (2014) construíram um estado da arte com trabalhos de pós-graduação strictu senso e trabalhos apresentados em eventos científicos da área, respectivamente, relacionados à Educação Estatística.

Observamos com os resultados dos autores que, no decorrer dos 14 anos investigados, há um número pequeno de pesquisas científicas relacionadas a área. O que nos mostra que existe uma lacuna a ser preenchida a respeito de tópicos de ensino de estatística.

Segundo Júnior e Vaz (2014), três conteúdos são principalmente abordados nos trabalhos analisados: Estatística, Probabilidade e Análise Combinatória. O estudo da utilização dos ponderadores estatísticos está principalmente relacionado com o tema Estatística, porém esse não foi um dos temas citados pelos autores, talvez por não ter representatividade entre os trabalhos analisados, o que nos leva a acreditar que esse é um tema pouco explorado em pesquisas de Educação Estatística, ou até mesmo inédito.

Já Bianchini (2013) apresenta um estado da arte com enfoque na prática pedagógica docente do ensino de estatística. O tema por nós abordado nesse relato está em correspondência com o eixo temático apresentado intitulado "Concepções, saberes e práticas docentes", o qual discorre sobre o conhecimento profissional dos professores de Matemática que atuam na Educação Básica e suas influências no processo de ensinoaprendizagem dos conceitos de Estatística. Tal autora aponta a necessidade de uma revisão curricular na formação inicial e continuada dos professores, no que diz respeito ao ensino da estatística. Porém, há apenas três pesquisas relacionadas ao eixo temático no período investigado, o que mostra uma necessidade de discutir ideias relacionadas a introdução de conceitos estatísticos por professores de Matemática na Educação Básica. 


\section{Proposta Metodológica para o Ensino de Média Estatística}

A partir de nossa Experiência Docente e Profissional com a Estatística, construímos uma discussão sobre o ensino de conceitos estatísticos, em especial a média estatística, na Educação Básica.

O primeiro contato, dos alunos, com o estudo da estatística acontece na matemática, durante a Educação Básica, mais precisamente com o cálculo da média, como pode ser observado nos PCN's da Educação Básica para a disciplina de Matemática (BRASIL, 2006). Este primeiro contato, em geral acontece se definindo média aritmética sem apresentar os ponderadores, logo, apresentando como a chamada "média simples". Muitas vezes até sem usar essa terminologia, o que pode ser observado em boa parte dos livros didáticos para a Educação Básica ${ }^{15}$, apenas apresentando a definição:

$$
\bar{x}=\frac{\sum_{i}^{n}=1^{x_{i}}}{n}=\frac{x_{1}+x_{2}+\cdots x_{n}}{n}
$$

Como os alunos passam muito tempo construindo a ideia de média apenas ligada a "média simples", a introdução muito posterior de ponderadores se torna uma dificuldade real observada em sala de aula, em diferentes níveis de ensino. A imagem construída pelos alunos de média simples faz com que, ao terem contato com situações onde os ponderadores são diferentes de 1 , tenham dificuldades em identificá-los e tendem a tentar reduzir as médias a serem sempre simples.

Uma proposta para melhor construir o conceito de média, $\bar{x}$, que apresentamos nesse artigo para professores que lecionam estatística, é construir o conceito a partir do caso geral, ressaltando a presença de ponderadores; e não a partir do caso particular, onde os ponderadores valem todos 1 .

Acreditamos que no ensino fundamental, é plausível que se apresente, prioritariamente, situações problema que envolvam somente a média simples. Mas situações simples que comecem a construir a presença de ponderadores diferentes de 1 , contribuiriam para desenvolver a capacidade cognitiva dos alunos de trabalhar posteriormente com ponderadores.

No ensino médio, sugerimos que seja mais apropriado construir o conceito de média já com a presença de ponderadores, apresentando o conceito de média como a Fórmula (2).

\footnotetext{
15 Optamos por não citar autores de livros didáticos, porém isso pode ser facilmente constatado com uma pequena pesquisa em alguns exemplares mais populares no Ensino.
} 


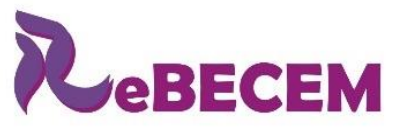

DOI: http://dx.doi.org/10.33238/ReBECEM.2019.v.3.n.2.22605

Nesse caso, a média simples é um caso particular onde os ponderadores valem todos 1 .

Mesmo que se apresente o caso particular de média aritmética simples como um item de estudo distinto da média aritmética ponderada, nossa crítica é, principalmente, sobre a ênfase que é dada a atividades envolvendo somente o caso particular na Educação Básica. Se assim fossem apresentadas, porém fossem desenvolvidas, no ensino médio, prioritariamente atividades envolvendo o caso geral, acreditamos que essa prática minimizaria as dificuldades posteriores apresentadas pelos alunos e profissionais em se trabalhar com ponderadores, e, por conseguinte, com Estatística.

Apresentamos a partir de nossa experiência profissional uma proposta para o ensino de Médias Estatísticas, porém o problema dos ponderadores também está presente nas demais quantidades estatísticas, principalmente, naquelas que nos retornam as estimativas pontuais, tais como: Totais, Mediana, Moda, Quantis, dentre outras, onde a utilização dos ponderadores é essencial em sua construção.

A seguir, de forma exemplificativa, estão as diferenças de cálculo de duas quantidades estatísticas, muito utilizadas, quando se fazem com e sem os pesos amostrais.

\section{Totais Estatísticos}

Sem os pesos é uma soma simples, o total é uma soma de todos os $N$ elementos de uma população.

$$
T=\sum_{i=1}^{n} x_{i}
$$

Com os pesos é uma soma ponderada, onde $w_{i}$ é o peso amostral associado ao $i$ ésimo elemento da amostra, que tem apenas $n$ unidades amostrais e que $n<N$.

$$
T=\sum_{i=1}^{n} w_{i} x_{i}
$$

No caso particular, em que $n=N$, temos que os pesos amostrais são todos iguais a um.

\subsection{Mediana}

A fórmula para se encontrar o valor central de uma população é dada após a ordenação de todos os elementos do conjunto populacional, e aí se tem 2 opções:

1. Se o conjunto tiver um número ímpar de elementos sua mediana será o seu elemento central, ou seja: 
DOI: http://dx.doi.org/10.33238/ReBECEM.2019.v.3.n.2.22605

$$
\tilde{x}=x_{\left(\frac{n+1}{2}\right)}
$$

2. Se o conjunto tiver um número par de elementos sua mediana será a média de seus 2 elementos centrais, ou seja:

$$
\tilde{x}=\frac{x\left(\frac{n}{2}\right)+x\left(\frac{n}{2}+1\right)}{2}
$$

Já quando se trabalha com amostras probabilísticas, temos que utilizar um conceito mais geral, pois os elementos da amostra não representam apenas a si, como também a outros elementos da população não selecionados. Desta forma, uma das maneiras de se calcular a mediana é após ordenar os elementos seguir os seguintes procedimentos:

1. Calcula-se a frequência do i-ésimo valor, $f_{i}=f\left(x_{(i)}\right)$,

$$
f_{i}=f\left(x_{(i)}\right)=\frac{w_{i}}{\sum_{i=1}^{n} w_{i}}
$$

onde, $w_{i}$ é o peso associado à estatística de ordem $x_{(i)}$.

2. Calcula-se a frequência acumulada dos valores menores ou iguais à $x_{(i)}, F_{i}=$ $\left(F(x)_{(i)}\right)$, onde:

$$
F_{i}=\left(F(x)_{(i)}\right)=\sum_{k=1}^{i} f_{k}
$$

3. A mediana será a primeira estatística de ordem, $x_{(i)}$, que satisfizer o seguinte critério:

$$
F_{i} \geq 0,5
$$

As fórmulas estatísticas anteriormente citadas podem ser encontradas nos livros de estatística, tais como: BOLFARINE e BUSSAB (2005), BUSSAB e MORETIN (2004) e COCHRAN (1977).

Outro lugar importante, onde deve-se aplicar os pesos amostrais, é na modelagem estatística, pois, na maioria dos casos, os modelos são construídos para serem aplicados a uma amostra de forma que possamos generalizar tais resultados para a população. Este é um dos principais equívocos produzidos por alunos de graduação, pós-graduação e profissionais formados.

Portanto, acreditamos que o trabalho com os ponderadores na educação básica e na graduação e com problemas que suscitem ao aluno um maior raciocínio lógicomatemático, minimizaria os entraves dos alunos destas etapas do ensino em trabalhar com estatística e principalmente com ponderadores. 


\section{Considerações finais}

Pode-se perceber no decorrer do texto que as formas de ensino matemático e estatístico são distintas. Segundo Echeveste et al., (2005) e Lopes (2010), os professores da Educação Básica devem abordar os conteúdos estatísticos de forma a suscitar no aluno a ideia da variabilidade, na qual a Educação Estatística está fundamentada. Assim, ao se estimar uma quantidade de interesse o aluno possa não só medir a quantidade, mas também a precisão da técnica utilizada, de forma a que se possam realizar análises realistas sobre o tema estudado ou escolher a técnica mais adequada à medição.

A abordagem dos autores citados anteriormente, juntamente com a nossa vivência profissional, corrobora com a nossa preocupação com o emprego dos pesos amostrais na construção das quantidades estatísticas, a fim de que o aluno consiga assimilar os conceitos estatísticos de forma prática e precisa. Pois, uma correta estimação das quantidades de interesse faz com que o aluno ou profissional possa interpretar de maneira correta o cotidiano à sua volta trazendo desta forma um melhor resultado para a sociedade.

\section{Referências}

BIANCHINI, D. F. Práticas pedagógicas em estatística dos anos iniciais: realidades e possibilidades. 2013. Dissertação (Mestrado em Educação nas Ciências), Universidade Regional do Noroeste do Estado do Rio Grande do Sul (UNIJUÍ), Ijuí, 2013.

BOLFARINE, H.; BUSSAB, W.O. Elementos de Amostragem. São Paulo: ABE - Projeto Fisher. Edgard Blücher. 2005.

BRASIL. Ministério da Educação. Orientações Educacionais Complementares aos Parâmetros Curriculares Nacionais (PCN+ Ensino Médio). Ciências da Natureza, Matemática e suas Tecnologias. Brasília: MEC, 2006.

BUSSAB, W.O.; MORETTIN, P.A. Estatística Básica. 5. ed. São Paulo: Saraiva. 2004.

COCHRAN, W.G. Sampling Techniques. 3. ed. Nova York: John Wiley \& Sons. 1977.

ECHEVESTE, S. et al. Educação Estatística: perspectivas e desafios. Acta Scientiae, Canoas/RS, v. 7, n.1, p. 103-109, jan./jun. 2005. Disponível em:

http://www.periodicos.ulbra.br/index.php/acta/article/view/191. Acesso em: 01 jun. 2019.

JÚNIOR, A. P. de O. J.; VAZ, T. H. Tendências da Pesquisa em Educação Estatística no Brasil de 2000 a 2013: Eventos Científicos. In: ENCONTRO NACIONAL DE PRÁTICA DE ENSINO, 17., 2014, Ceará. Anais... Ceará: EdUECE, 2014. p. 1-5. Disponível em: http://www.uece.br/endipe2014/ebooks/livro2/TEND\%C3\%8ANCIAS\%20DA\%20PESQUISA $\% 20$ EM $\% 20$ EDUCA $\%$ C $3 \% 87 \%$ C $3 \% 830 \% 20$ ESTAT $\%$ C3\%8DSTICA $\% 20$ NO $\% 20$ BRASIL $\%$ 20DE\%202000\%20A\%202013\%20EVENTOS\%20CIENT\%C3\%8DFICOS.pdf. Acesso em 10 ago. 2019. 


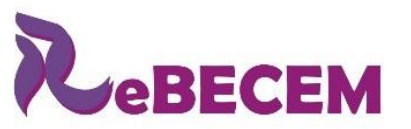

Revista Brasileira de Educação em

Ciências e Educação Matemática

DOI: http://dx.doi.org/10.33238/ReBECEM.2019.v.3.n.2.22605

LOPES, C. E. A Educação Estatística no Currículo de Matemática: Um desafio teórico. In:

REUNIÃO ANUAL - ANPED, 33, 2010, Caxambu. Anais... Caxambu: ANPED, 2010. p. 1-15. Disponível em:

http://33reuniao.anped.org.br/33encontro/app/webroot/files/file/Trabalhos\%20em\%20PDF/GT1 9-6836--Int.pdf . Acesso em: 01 jun. 2019.

Recebido em: 25 de junho de 2019.

Aceito em: 16 de agosto de 2019. 